\title{
FORECASTING ELECTRICITY SUPPLIED IN TURKEY USING HOLT- 'WINTERS' MULTIPLICATIVE METHOD AND ARTIFICIAL NEURAL NETWORK (ANN) MODELS
}

\author{
SITI NOR ZULAIKA AMRAN AND NORIZAN MOHAMED*
}

Faculty of Ocean Engineering Technology and Informatics, Universiti Malaysia Terengganu, 21030 Kuala Nerus, Terengganu, Malaysia.

*Corresponding author: norizan@umt.edu.my

http://doi.org/10.46754/umtjur.2021.07.014

\begin{abstract}
Electricity is one of the most essential necessities in today's world and has an important role for the development of societies and economics. The need for electricity is expanding continuously due to increasing population, urbanization and industrialization. Hence, the purpose of this study was to develop the best model for forecasting electricity supplied in Turkey by applying the multiplicative Holt-Winters method and multilayer feed-forward neural network model. The monthly electricity supplied in Turkey from January 2000 until December 2019 were obtained from monthly electricity statistics report presented by the International Energy Agency (EIA). The data were divided into two sets comprising in-sample data from January 2000 until December 2015 and out-sample data from January 2016 to December 2019. The multiplicative Holt-Winters was used since the electricity supplied in Turkey exhibit trend and seasonal gave the out-sample forecast of 3.6990. The best multilayer feed forward neural network (MFFNN) model with three input lag variable, one hidden node, one output node, sigmoid transfer function in hidden layer and linear transfer function in output layer gave the out-sample forecast of 2.1483 . Hence it can be concluded that, the multilayer feedforward neural network model is more accurate than multiplicative Holt-Winters method to forecast the electricity supplied in Turkey.
\end{abstract}

Keywords: Electricity supplied in Turkey, multiplicative Holt-Winters, exponential smoothing, multilayer feed forward Neural network (MFFNN)

\section{Introduction}

Electricity is important for industrialdevelopment and activity. Electricity has become a big part of our daily life. Our daily routines depend heavily on the use of electricity whether it is at home, school, workplace, hospital or at the shopping center. Therefore, providing sufficient electrical energy is necessary for people in the society because it affects their life directly or indirectly since it has been found that adequate electrical supply is a key requirement for cultural, social and economic development in societies (White, 1943; Cottrell, 1955; Tainter, 1990).

Overall energy demand is increasing because of the population development and technological sophistication and it is anticipated to arrive more than twice the current level by 2050 . The less access to electricity, the less will be the economic and human improvement of nations. So, some researchers used the forecasting method to analyz the data of electricity supplied to make sure all the people get sufficient electricity. It is important to forecast the electricity supply to avoid bottlenecks, shortages and surpluses of electricity. The monthly electricity supplied in Turkey will be tested and reviewed in this study.

The paper aims to predict the electricity supplied in Turkey using two methods, which are exponential smoothing and artificial neural networks (ANN). In this study, the following objectives were executed; the first objective is to apply the exponential smoothing and ANN into the data of electricity supplied in Turkey. 
The second objective is to examine the seasonal variations and trends of the electricity supplied in Turkey and the last objective is to choose the best method between exponential smoothing and ANN models in predicting the electricity supplied within Turkey.

Data of electricity supplied in Turkey was used in this study. Electricity supplied data in Turkey was obtained from the monthly electricity statistics report presented by the International Energy Agency (EIA). The data involved is the data of monthly electricity supplied in Turkey. It included 240 observations within the period starting from January 2000 until December 2019.

\section{Research of electricity}

Gulay (2019) proposed predicting the electricity generation within South Africa. The quarterly total of electricity generation within South Africa from January 1985, Quarter 1 to September 2017, Quarter 3 were used which are provided from the internet page of Federal Reserve Economic Data. The R program was used to forecast the total electricity generation in this paper. The comparison between hybrid predicting and time series method were identified. As the result, it was found that hybrid predicting which was Artificial Neural Network based on the multiplicative Holt-Winters was more accurate to predict the total electricity generation within South Africa.

Swaroop and Abdulqader (2012) proposed forecasting the electricity load by using ANN method. The actual data of electricity load in a specific district known as Al Batinah of Oman were obtained from the Mazoon Electrical Company, Oman for the year 2007 to 2009. This data was run using neural networks method to predict the forthcoming. The data from years 2007 to 2009 were used as training to forecast the output for the year 2010. The 40 multilayer perceptron (MLP) network model was used in this study and the result showed that MLP network achieved the best performance and prediction accuracy. The mean absolute error
(MAE) was computed to evaluate the forecasting reliabilities and the values found for MAE was $2.64 \%$ which represents an accurate accuracy. It was concluded that the ANN model performed better with small error.

\section{Research by using exponential smoothing}

Lim and McAleer (2001) forecasted the quarterly worldwide visitors to Australia using exponential smoothing techniques. The data of present and previous advent from Singapore, Malaysia, and Hong Kong were compared using various exponential smoothing methods. The result showed that for entry from Hong Kong and Singapore, multiplicative seasonality of Holt-Winters outperformed the other methods while the additive seasonality of Holt-Winters performed more accurate for entry from Malaysia. As conclusion, the additive of HoltWinters and multiplicative of Holt-Winters gave lower post-sample forecast errors in both levels and logarithms in this study.

Siregar et al. (2017) proposed forecasting the production of palm oil using the exponential smoothing methods. In this paper, the single exponential smoothing, Holt's method and HoltWinters' method were compared based on error measurement values such as mean absolute percentage error (MAPE), mean absolute error (MAE) and Root mean square error (RMSE). The results showed that, the MAPE, MAE and RMSE of additive Holt-Winters was less than Holt's method and multiplicative Holt-Winters for seasonal data on the production data of Seri Menanti Plantations.

Rusyana Asep et al. (2016) applied Holt's method and Holt-Winters' method in forecasting the total passengers at the Sultan Iskandar Muda International Airport who were from Acheh. The mean square error (MSE), mean absolute percentage error (MAPE) mean absolute error (MAE) and mean percentage error (MPE) were used as an error measurement in this paper. As a result, they found that, the triple exponential smoothing for multiplicative Holt-Winters was more accurate compared to other methods to 
predict the total of local entry with MAE, MSE, MPE and MAPE of 1230, 3215248, 0.18 and 5.41 respectively. While for the forecasting of the number of local departure, the triple exponential smoothing for multiplicative model with MAE, MSE, MPE and MAPE of 1220, 2671955, 0.02, and 5.12 respectively was the most accurate.

\section{Research by using ANN Method}

Hamdan et al. (2018) proposed forecasting the weather in Palestine using artificial neural networks (ANN) models. The data set was acquired from weather records of the Palestinian Meteorological Department (PMD) in Palestine for 3 years starting from 2013 to 2015. In this study, the Multilayer Perceptron Feed-Forward Neural Networks with Backpropagation Algorithm (MLPFFNNBP) was used. For the result, they found MLPFFNNBP model produced a better result of forecasting than using other classical methods of forecasting with an appropriate number of hidden nodes.

Rodrigues (2014) applied feedforward artificial neural network (ANN) models in forecasting the energy use and load predicting. In this research, a dataset of usage reports was used, registered in 93 different residences, and consisted of weekdays and weekends in Lisbon, Portugal, from the period of February 2000 to July 2001. A feedforward neural network consists of three-layered trained with Levenberg-Marquardt was used in this paper. As a conclusion, it was found that the ANN models produced a good forecasting for household electricity consumption and load profile.

Fauziah et al. (2017) proposed forecasting the sugar cost by using Holt's method and artificial neural network (ANN). The mean data of sugar cost was taken from 7 markets in Depok within a week. The Holt's method and neural network was compared. The Mean Squared Error (MSE) value by using Holt exponential smoothing method was 403282 whereas the ANN was 15341.2. Then, the Mean Absolute Percentage Error (MAPE) using Holt exponential smoothing method was 1.12 and
ANN was 0.74 . Hence, the ANN model was the best in forecasting the mean price of sugar $/ \mathrm{kg}$ in Depok.

\section{Methodology}

This part discusses the methods used in conducting the electricity supplied forecasting in Turkey. Two methods which are the Exponential Smoothing and Artificial Neural Network were applied.

\section{Exponential Smoothing Method Approach}

Exponential smoothing is a univariate data forecast approach for time series that supports trend or seasonal portion. Robert Goodell Brown suggested the method of exponential smoothing in 1956, then extended by Charles C. Holt in 1957. To predict the future, the exponential smoothing flatten previous data from a time series. The three kinds of exponential smoothing are the simple exponential smoothing (SES), double exponential smoothing (DES) or Holt method and triple exponential smoothing or the Holt-Winters method. Three equations are involved in the Holt-Winters method which each connected to level, trend and seasonality (Christo et al., 2013). There are two forms of the Holt-Winters method; multiplicative and additive.

\section{Multiplicative Holt-Winters}

For level, trend and seasonal changes towards forecast, multiplicative Holt-Winters' calculates exponentially smoothed values. Then, multiplicative with seasonal approach increases the trended seasonality prediction, generating multiplicative forecast for Holt-Winters. This approach is suitable for increases over time data with trend and seasonality. It contributes to a curved forecast which imitates the data's seasonal changes. It is considered as the best model to forecast a time series that can be shown by:

$$
y_{t}=\left(B_{0}+B_{1} t\right) S N_{t} I R_{t}
$$


Where $S N_{t}$ is a seasonal pattern and $I R_{t}$ is an irregular component. There are three equations involved in Multiplicative Holt-Winters which are:

Level estimation : $\ell_{t}=\alpha\left(y_{t} / s n_{T-L}\right)+(1-\alpha)\left(\ell_{T-1}+b_{T-1}\right)$

Trend estimation: $b_{t}=\gamma\left(\ell_{t}-\ell_{t-1}\right)+(1-\gamma) b_{T-1}$,

Seasonal estimation: $s n_{t}=\pi\left(y_{t} / \ell_{T}\right)+(1-0) s n_{T-L}$

where smoothing constants, $\alpha, \gamma$, and $\delta$ from 0 and 1 and $L$ is the number of periods per year ( $\mathrm{L}=12$ for monthly data, and $\mathrm{L}=4$ for quarterly data). The value of prediction at time $\mathrm{T}$ for $y_{T+p}$ is given by $\hat{y}_{T+p}(T)-\left(\ell_{t}+p b_{T}\right) s n_{T+p-L}$ where $(\mathrm{p}=1,2,3, \ldots)$.

\section{Artificial Neural Network (ANN) Model Approach}

One form of artificial intelligence technique that mimics human brain activity is the artificial neural network (ANN) (Haykin, 2009). Artificial neural network is a computer model which focused on biologic neural network architectures and functions. The basic structure of an ANN consists of artificial neurons organized into layers that are equivalent to biological neurons in the human brain. The common ANN structure consist of an input layer, one or more hidden layers, and an output layer. In general, there are two types of ANN; feedforward and recurrent neural network. One of the simplest type of ANN is the feedforward artificial neural network, where data or input moves in one direction and no loops exist. From the first tier downwards, data travels in just one direction before it reaches the output node. In addition, a recurrent neural network (RNN) is the form of artificial neural network where an exact layer's output is stored and returned to the input.

\section{Multilayer Feed Forward Neural Network (MFFNN)}

Multilayer networks consist of at least one hidden layer between the input and output layers, as opposed to a single-layer network. The role of hidden neurons, according to Haykin, is to interfere in some useful way between the external input and the network output. The presence of one or more hidden layers allows higher-order statistics to be collected by the network. It is called multilayer since it contain processing units layer (hidden units) in addition to the output units and feedforward due to the output from single layer of neurons feeds into the following layer of neurons. An input layer, one or more hidden layers, and an output layer constitute the multi-layer feedforward neural network.

The inputs are fed inside the units concurrently and the input layer is formed. These inputs move via the input layer and are measured and fed simultaneously to the next layer called the hidden layer. It is possible for the outputs of a hidden layer to be the input for a different hidden layer. The multilayer feed forward neural network (MFFNN) model architecture with a hidden layer, $\mathrm{N}$ input nodes, $\mathrm{H}$ hidden nodes and an output node is shown in Figure 1. 


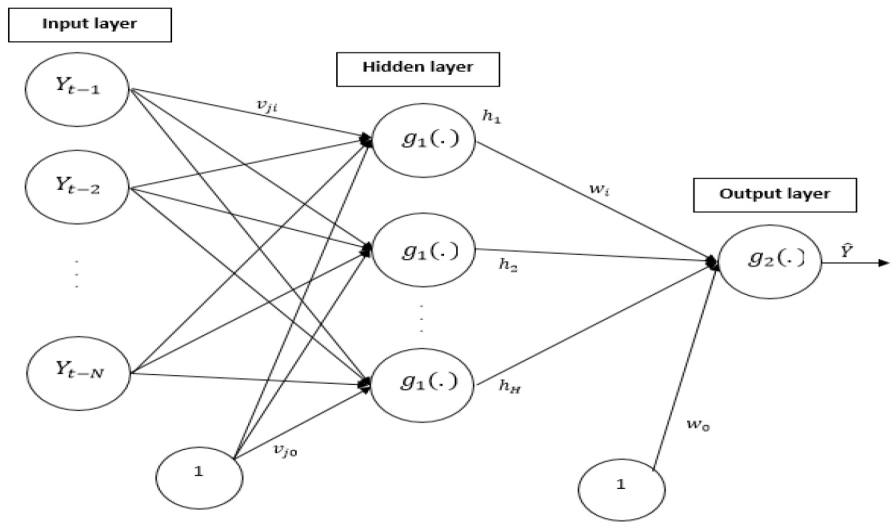

Figure 1: The architecture of MFFNN model with a hidden layer, $\mathrm{N}$ input nodes, $\mathrm{H}$ hidden nodes and an output node

The connection between the neurons are represented as weight. Each neuron consists of a function of summing and activation function. The value $\hat{Y}$ for the feedforward network with $\mathrm{N}$ inputs nodes, $\mathrm{H}$ hidden nodes and an output node is :

$$
\hat{\mathrm{Y}}=g_{2}\left(\sum_{j=1}^{H} w_{j} h_{j . t}+w_{0}\right)
$$

Here $w_{j}$ is an output weight between hidden node $\mathrm{j}$ and output node, $w_{0}$ is output node bias, and $g_{2}$ is an activation function. The hidden nodes $h_{j}, j=1, \ldots, H$ values are shown below:

$$
h_{j, t}=g_{1}\left(\sum_{t=1}^{N} v_{j i} y_{t, i}+v_{j 0}\right) j=1,2, \cdots H
$$

where, $v_{j i}$ is the input weight between the input node $i$ and hidden node $j, v_{j 0}$ is the hidden node $\mathrm{j}$ bias, $y_{t, i}$ are the lag variables where $\left(y_{t, i}\right.$ $\left., \ldots, y_{t, N}\right)$ are $\left(y_{t-i}, \ldots, y_{t-N}\right), i=1,2, \ldots, N$. and $g_{1}$ is a function for activation.

\section{Evaluating Model Performance}

Tests were carried out to analyze the model precision after estimating the electricity supplied in Turkey. The Mean Absolute Percentage Error (MAPE) has been used to assess the precision of estimated values determined by the methods used. The accuracy of MAPE measuments is defined as follows:

$$
\mathrm{MAPE}=\mathrm{MAPE}=\frac{1}{n} \sum_{t=1}^{n} \frac{\left|y_{t}-y_{t}\right|}{y_{t}} \times 100
$$

whereas $\hat{y}_{t}$ is the forecast value at point $t ; \hat{y}_{t}$ is the original value at point $t ; n$ is the number of observations.

The level of accuracy of MAPE values as presented in Table 1 below (Lewis, 1982).

Table 1: The level of forecasting based on the values of MAPE

\begin{tabular}{cc}
\hline MAPE & Explanation \\
\hline$<10 \%$ & High accuracy forecasts \\
$10 \%-20 \%$ & Good forecasting \\
$20 \%-50 \%$ & Reasonable forecasting \\
$>50 \%$ & Inaccurate forecasts \\
\hline
\end{tabular}

\section{Results and Discussion}

This part discusses the prediction results of electricity supplied in Turkey when we applied the Exponential Smoothing and the Artificial Neural Network models. As for research purpose, secondary data of electricity supplied in Turkey was used. The data comprise findings of the period from January 2000 until October 2019 that includes 240 samples of data. The data samples were divided into in-sample 
and out-sample. Between January 2000 and December 2015, there are 192 data samples in the in-sample data set, while the out-sample data set comprised a period from January 2016 to December 2019 with 48 data samples.

\section{Exponential Smoothing Method}

Figure 2 is a time series plot for in-sample data of electricity supplies in Turkey by using
Minitab software. The time series exhibit nonstationary based on the time series plot in Figure 2. Therefore, differencing was carried out to make it stationary. Figure 3 below shows the time series plot after first difference for insample data of electricity supplies in Turkey while Figure 4 below shows the autocorrelation plot after first difference for in sample-data of electricity supplies in Turkey.

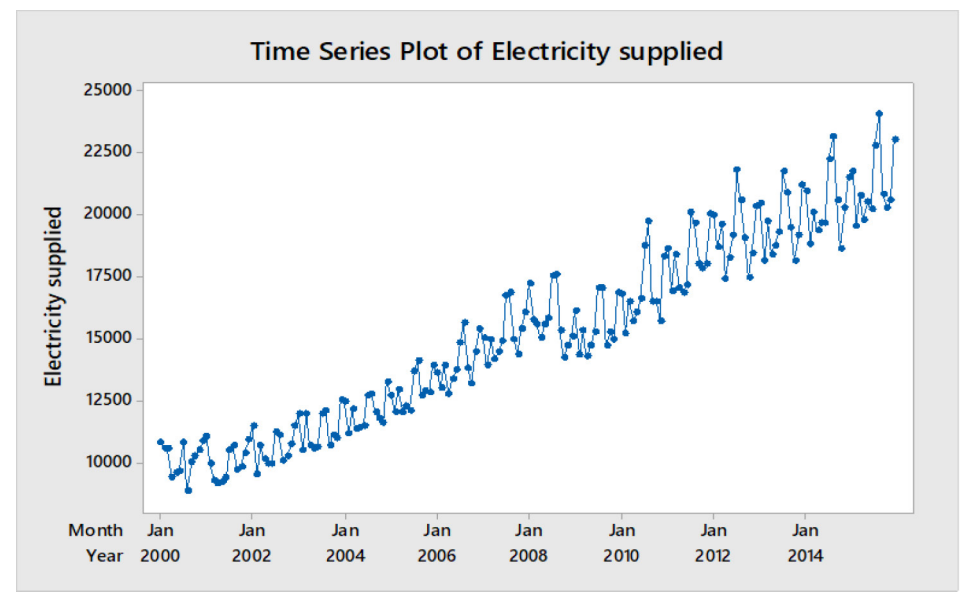

Figure 2: Time series plot for in-sample data of electricity supplies in Turkey



Figure 3: Time series plot after first difference for in-sample data of electricity supplies in Turkey. 


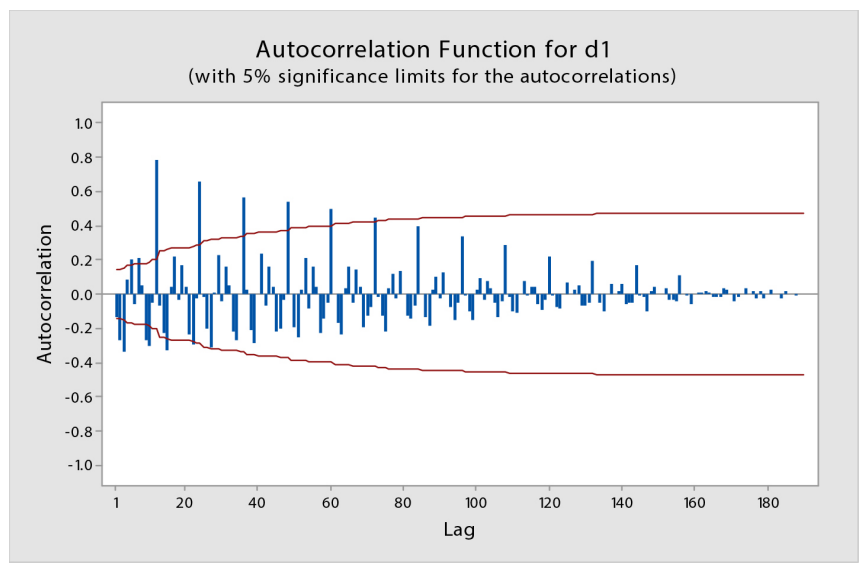

Figure 4: Autocorrelation plot after first difference for in sample-data of electricity supplies in Turkey.

Based on Figure 2 also, the in-sample data of electricity supplies in Turkey were experiencing an upward trend from Jan 2000 to Dec 2015. In addition, from Figure 4, the in-sample data of electricity supplies in Turkey indicate the presence of seasonality which is monthly seasonality with length $12, \mathrm{~s}=12$. Therefore, multiplicative Holt-Winters was used as the data exhibit trend and seasonal patterns.

\section{Multiplicative Holt-Winters}

First, initialization parameters $\alpha, \gamma$ and $\delta$ which can minimize error was specified to obtain the Winter's exponential smoothing equation. Minitab software is used to find the best value of smoothing constants $\alpha, \gamma$ and $\delta$. By trial and error approach, the optimal smoothing constant obtained for the level is $\alpha=0.50$, the smoothing constant of trend pattern $\gamma=0.01$ and seasonality $\delta=0.01$. The smoothing constant value produces the minimum error, with a MAPE value of 3 . According to the result of smoothing constant value, the winter's exponential smoothing equation with multiplicative model for forecasting the electricity supplies in Turkey can be written as below.

Level component:

$\mu_{n}=0.5\left(Y_{n} / s_{n-12}\right)+0.5\left(\mu_{n-1}+T_{n-1}\right)$

Trend component:

$T_{n}=0.01\left(\mu_{n}-\mu_{n-1}\right)+0.99\left(T_{n-1}\right)$

Seasonality component:

$S_{n}=0.01\left(Y_{n} / \mu_{n}\right)+0.99\left(S_{n-1}\right.$ :

The m-step ahead forecast is calculated as:

$\hat{\mathrm{y}}_{n+m}=\left(\mu_{n}+T_{n} m\right) S_{n-12+m}$

From the optimum smoothing constants obtained, a four-year forecast was performed. Figure 5 showed the actual and forecast values of electricity supplies in Turkey from January 2016 to December 2019 by using multiplicative Holt-Winters with constant $\mathrm{a}=0.50, \quad \mathrm{~g}=0.01$ and $\mathrm{d}=0.01$. 


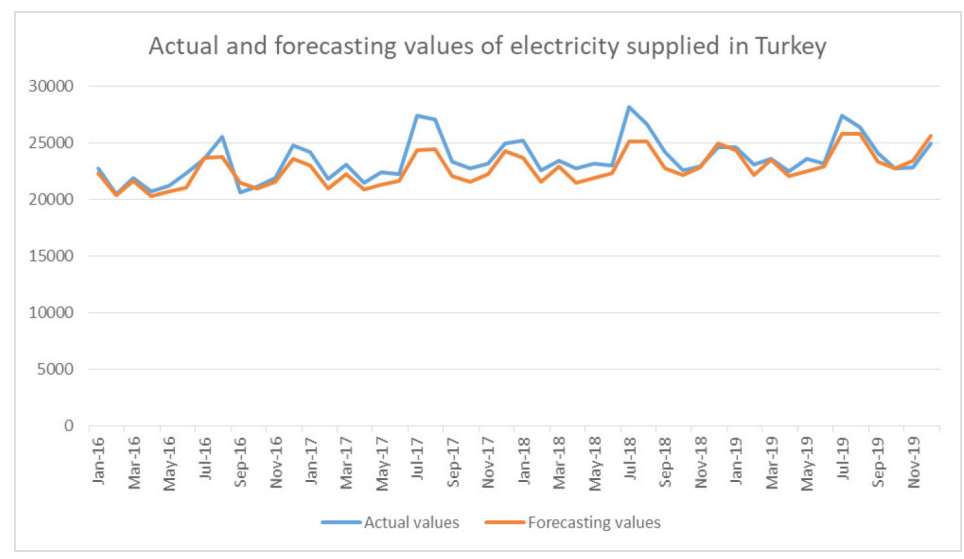

Figure 5: Actual and forecast vales from January 2016 to December 2019 of Multiplicative Holt-Winters method

The MAPE using $\alpha=0.50, \gamma=0.01$ and as variables of the input to find the best hidden $\delta=0.01$ for in-sample dataset and out-sample dataset of electricity supplies within Turkey are shown in Table 2. nodes number and the total of hidden nodes is increase from 1 until 10 . Table 3 showed the MAPE of hidden nodes from 1 to 10 nodes.

Table 2: The MAPE for in-sample dataset and outsample dataset of electricity supplies in Turkey

\begin{tabular}{cc}
\hline DATA & MAPE \\
\hline In-sample & 3 \\
(January 2000 until December 2015) & \\
Out-sample & \\
(January 2016 to December 2019) & 3.6990 \\
\hline
\end{tabular}

\section{Multilayer Feed-Forward Neural Network} (MFFNN)

In this paper, the best training algorithm is Levenberg-Marquardt, the transfer function within hidden layer is the log-sigmoid function and the transfer function within output layer is the linear transfer function. Input nodes consisting of a lag variable are chosen based on the ARIMA model for electricity supplied in Turkey. The ARIMA $(0,1,1)(0,1,0)_{12}$ which can be written as $y_{t}-y_{t-12}-y_{t-1}+y_{t-13}=(1-0.6006 \mathrm{~B}) \varepsilon_{t}$ was identified as the best of ARIMA model. Hence the input nodes are 3 lag variables which are $y_{t-12}-y_{t-1}$ and $y_{t-13}$.

All lag variables in the ARIMA $(0,1,1)(0$, $1,0)_{12}$ model which are $y_{t-12}, y_{t-1}$ and $y_{t-13}$ are used
Table 3: The number of hidden nodes

\begin{tabular}{ccc}
\hline Hidden Nodes & MAPE Training & MAPE Testing \\
\hline $\mathbf{1}$ & $\mathbf{2 . 8 4 5 4}$ & $\mathbf{2 . 1 4 8 3}$ \\
2 & 2.7355 & 2.2072 \\
3 & 2.6426 & 2.8795 \\
4 & 2.5509 & 3.1162 \\
5 & 2.4849 & 3.4764 \\
6 & 2.3784 & 3.5188 \\
7 & 2.2235 & 3.7038 \\
8 & 2.1808 & 4.4000 \\
9 & 2.0786 & 5.3312 \\
10 & 1.8915 & 5.4000 \\
\hline
\end{tabular}

By using one node as the hidden node number (refer to Table 3), the log-sigmoid function within the hidden layer and the linear transfer function within the output layer, we try to determine the best lag variables number by combining the lag variables. Table 4 shows the results of MAPE training and MAPE testing for combination of lag variables ranging from one lag variable to three lag variables. 
Table 4: The result of combination of input lag variables

\begin{tabular}{cccc}
\hline $\mathbf{n}$ & $\begin{array}{c}\text { Input } \\
\text { Variables }\end{array}$ & $\begin{array}{c}\text { MAPE } \\
\text { Training }\end{array}$ & $\begin{array}{c}\text { MAPE } \\
\text { Testing }\end{array}$ \\
\hline 1 & $y_{t-1}$ & 5.9196 & 8.4391 \\
& $y_{t-12}$ & $\mathbf{3 . 4 9 1 7}$ & $\mathbf{2 . 5 4 8 7}$ \\
& $y_{t-13}$ & 6.5294 & 8.0645 \\
\multirow{2}{*}{2} & $y_{t-12}, y_{t-1}$ & $\mathbf{3 . 2 8 6 1}$ & $\mathbf{2 . 5 4 2 4}$ \\
& $y_{t-12}, y_{t-13}$ & 3.4809 & 2.5385 \\
3 & $y_{t-12}, y_{t-1}, y_{t-13}$ & $\mathbf{2 . 8 4 5 4}$ & $\mathbf{2 . 1 4 8 3}$ \\
\hline
\end{tabular}

The best performances of lag variables number ranging from one to three together with the best combination of lag variables is summarized in Table 5. The result showed that three nodes is the best number of input nodes.
The inputs variables are the lag variables combination $y_{t-12}, y_{t-1}$ and $y_{t-13}$.

Table 5: Summary of input lag variables

\begin{tabular}{cccc}
\hline $\mathrm{n}$ & Input Variables & MAPE & MAPE \\
& & Training & Testing \\
\hline 1 & $y_{t-12}$ & 3.4917 & 2.5487 \\
2 & $y_{t-12}, y_{t-1}$ & 3.2861 & 2.5424 \\
$\mathbf{3}$ & $y_{t-12}, y_{t-1}, y_{t-13}$ & $\mathbf{2 . 8 4 5 4}$ & $\mathbf{2 . 1 4 8 3}$ \\
\hline
\end{tabular}

Referring to Table 5, the best combination of input lag variables are $y_{t-12}, y_{t-1}$ and $y_{t-13}$. We then present the multilayer feed-forward neural network (MFFNN) architecture by three input of lag variables, a hidden node, an output node, sigmoid transfer function within hidden layer and linear transfer function within output layer as shown in Figure 6.



Input layer Hidden layer Output layer

Figure 6: MFFNN model architecture by three of input lag variables, a hidden node, an output node, sigmoid transfer function within hidden layer and linear transfer function within output layer

\section{Discussion}

The results of MAPE values for both methods are shown in Table 6. The MAPE out sample values for the MFFNN model performed better than Multiplicative Holt-Winters model of 3.0000 and 3.6990 respectively. This proves that the Artificial Neural Network (ANN) method specifically the MFFNN model is very effective and appropriate method to forecast the electricity supplied in Turkey.
Table 6: Comparison between Multiplicative Holt-Winters and MFFNN from MAPE values.

\begin{tabular}{ccc}
\hline Model & MAPE Training & MAPE Testing \\
\hline $\begin{array}{c}\text { Multiplicative } \\
\text { Holt-Winters } \\
\text { MFFNN }\end{array}$ & 3.0000 & 3.6990 \\
\hline
\end{tabular}




\section{Conclusion}

In this paper, the data of monthly electricity supplies in Turkey was obtained from monthly electricity statistics report that presented by International Energy Agency (EIA). After data undergoes the first differential method, it is found that the data has seasonal and trend components. Therefore, it is evident that the Multiplicative Holt-Winters should be used for this study. For ANN, the MFFNN model is selected. Various processes have been made to find inputs, hidden layers, and outputs using MATLAB. This process will produce an output which is the best model for the ANN method. The MAPE value of the data for out samples were compared for both methods which are Multiplicative Holt-Winters and ANN. The results in the study show that the value of MAPE out samples or Multiplicative Holt-Winters is 3.6990 while for MFFNN is 2.1483. This decision clearly shows that the MFFNN is the best model as compared to Multiplicative HoltWinters because it has lower MAPE values.

\section{Acknowledgements}

The authors would like to thank the Faculty of Ocean Engineering, Technology and Informatics, Universiti Malaysia Terengganu.

\section{References}

Christo E., Ferreira M. and Alonso K. 2013. 'Use of Statistical Control for Improved Demand Forecasting', Computational Intelligence and 11th Brazilian Congress on Computational Intelligence (BRICS-CCI \& CBIC), 2013 BRICS Congress on. IEEE.

Cottrell, W. F. (1955). Energy and society: The relationship between energy, social change, and economic development. McGraw-Hill.
Fauziah, F. N., Gunaryati, A., \& Sari, R. T. K. (2017). Comparison forecasting with double exponential smoothing and artificial neural network to predict the price of sugar. International Journal of Simulation-Systems, Science \& Technology, 18(4), 13-1.

Gulay, E. (2019). Forecasting the total electricity production in South Africa: Comparative analysis to improve the predictive modelling accuracy. AIMS Energy, 7(1), 88.

Hamdan, I., Awad, M., \& Sabbah, W. (2018). Short-Term Forecasting Of Weather Conditions in Palestine Using Artificial Neural Networks. Journal of Theoretical \& Applied Information Technology, 96(9).

Haykin S. S. (2009). Neural networks and learning machines, Prentice Hall/Pearson, NY.

Lewis, C.D. (1982). Industrial and business forecasting methods. London: Butterworths.

Lim, C., \& McAleer, M. (2001). Forecasting tourist arrivals. Annals of Tourism Research, 28(4), 965977.

Rodrigues, F., Cardeira, C., \& Calado, J. M. F. (2014). The daily and hourly energy consumption and load forecasting using artificial neural network method: a case study using a set of 93 households in Portugal. Energy Procedia, 62, 220-229. 
Rusyana Asep, Nurhasanah, Oktaviana, Swaroop, R., \& Abdulqader, H. A. Maulina \& Hasan, Amiruddin. (2012). Load forecasting for power (2016). Forecasting Passenger by Using Holt's Exponential system planning and operation Smoothing and Winter's Exponential Smoothing.

Siregar, B., Butar-Butar, I. A., Rahmat, using artificial neural network At Al Batinah Region Oman. Journal of Engineering Science and Technology, 7(4), 498-504.

R. F., Andayani, U., \& Fahmi, F. Tainter, J. (1990). The collapse of (2017, January). Comparison of exponential smoothing methods in forecasting palm oil real production. In Journal of Physics: Conference Series 801(1), 012004. IOP Publishing. complex societies. Cambridge University Press.

White, L. A. (1943). Energy and the evolution of culture. American anthropologist, 45(3), 335-356. 
